\title{
Ultra-flexible multiband terahertz metamaterial absorber for conformal geometry applications
}

\author{
Riad Yahiaoui, ${ }^{1, *}$ Jean Paul Guillet, ${ }^{2}$ Frédérick de Miollis, ${ }^{2}$ and Patrick Mounaix ${ }^{2}$ \\ ${ }^{1}$ XLIM, Univ. Limoges, CNRS, UMR 6172, 7 rue Jules Vallès, 19100 Brive, France \\ ${ }^{2}$ LOMA, Univ. Bordeaux1, CNRS, UMR 5798, 351 Cours de la Libération, 33405 Talence Cedex, France \\ *Corresponding author: riad.yahiaoui@unilim.fr
}

\begin{abstract}
Standard optical lithography relying on clean room and microelectronic facilities is used to fabricate a thin flexible metamaterial absorber, designed to operate at submillimeter wavelengths over the $0.11 \mathrm{THz}$ frequency band. Large terahertz absorption has been demonstrated numerically and through experimental measurements with a maximum level of about $80 \%$. We put emphasis in this present work on the use of single sized "meta cells" to achieve multiple absorption peaks. Furthermore, the use of a thin flexible dielectric spacer makes it promising for stealth technology applications in order to disguise objects and make them less visible to radar and other detection methods. (C) 2013 Optical Society of America

OCIS codes: (140.3490) Lasers, distributed feedback; (060.2420) Fibers, polarization maintaining; (060.3735) Fiber Bragg gratings; (060.2370) Fiber optics sensors.
\end{abstract}

Metamaterials with their unusual characteristics have revolutionized the world of physics. Considered as artificial materials based on metallic particles embedded in a host dielectric medium, metamaterials have attracted considerable interest from the scientific community for more than a decade. The concept and elementary theory of metamaterials dates back to the late 1960s, with the pioneering work of Veselago related to the negative refraction [1]. Considered one of the most fascinating properties of metamaterials, negative refraction was demonstrated experimentally in [2]. The authors, inspired from theoretical works related in [3,4], proposed a composite structure that combines continuous wires (CWs) and split-ring resonators (SRRs). Using an appropriate polarization of the incident electromagnetic wave ( $E$ parallel to the CWs and $H$ normal to the SRRs planes), this gives rise simultaneously to negative permittivity and negative permeability and thus to a negative refractive index at the desired frequency. Recently, another property of the metamaterials, namely, the near-perfect absorption involved in many well-known applications such as bolometer [5], photodetector [6], and hyperspectral imaging [7], has also aroused great enthusiasm within the community of metamaterials [ $\underline{8}-\underline{10}]$.

The electromagnetic effective properties $\varepsilon_{\text {eff }}$ (dielectric permittivity) and $\mu_{\text {eff }}$ (magnetic permeability) of a metamaterial-based absorber can be tailored by adjusting judiciously the design of the elementary building blocks of the structure in order to simultaneously achieve better impedance $z_{\text {eff }}$ matching to free space and a large imaginary part of the refractive index $n_{\text {eff }}$. The propagation of the incident EM wave is then highly attenuated within the structure. The transmittance is minimized, as well as the reflectance, thus giving rise to a large level of absorption. In other words, the concept of absorption consists to adjust the constitutive effective parameters of the structure ( $\varepsilon_{\text {eff }}$ and $\left.\mu_{\text {eff }}\right)$ in order to have better control on the propagative effective parameters $\left(z_{\text {eff }}\right.$ and $\left.n_{\text {eff }}\right)$. Due to inherent resonant behaviors of metamaterials, their exotic electromagnetic properties are achieved over narrow frequency ranges. Nevertheless, a variety of metamaterial structures have been demonstrated recently with specific characteristics over broad frequency ranges [11-15]. Previous works based on the use of different scales of resonators and multilayer approaches have demonstrated the ability to achieve multiband [16,17] and broadband metamaterial absorbers [18]. In this Letter, we present the design, fabrication, and experimental characterizations of a multiband (three main peaks) ultraflexible metamaterial absorber operating in the $0.1-1 \mathrm{THz}$ frequency range. The novelty brought by our investigated structure is its simplicity in manufacturing, thus focusing on the use of single-sized resonators. Furthermore, the use of a thin-flexible dielectric spacer makes our structure promising for conformal geometry applications. First, we describe the proposed metamaterial absorber. Second, numerical simulations using finite element method and experiments using terahertz time domain spectroscopy were carried out. The measured results agree well with the numerical predictions. Finally, an alternative double-layered structure is proposed in order to enhance the level of absorption.

The proposed metamaterial absorber is shown in Fig. 1. It consists of $200 \mathrm{~nm}$ thick aluminum patterns printed on the top side of $50 \mu \mathrm{m}$ thick dielectric substrate, while its bottom side is entirely coated with $200 \mathrm{~nm}$ thick aluminum to prevent any transmission through the structure. The elementary cell of our metamaterial absorber is represented in the inset (top left) of Fig. 1, with appropriate polarizations of electric and magnetic fields. It can be regarded as a combination of an inner cut wire in the direction of the incident electric field $E$ and an outer two-gap SRR. Other works that take advantage of the remarkable properties of the SRRs in the terahertz regime have been reported in $[19,20]$. The relevant geometrical dimensions of the unit cell are $a=b=300 \mu \mathrm{m} ; c=$ $50 \mu \mathrm{m}, d=100 \mu \mathrm{m} ; e=20 \mu \mathrm{m} ; f=250 \mu \mathrm{m} ; g=50 \mu \mathrm{m}$; $l=150 \mu \mathrm{m} ; t=50 \mu \mathrm{m} ; t g=200 \mathrm{~nm}$. The commercial dielectric substrate Kapton, which has a thickness $t$ of $50 \mu \mathrm{m}$, a relative dielectric permittivity $\varepsilon_{r} \sim 3$, and a moderate loss tangent $\tan \delta \sim 0.05$, up to $2 \mathrm{THz}$ (controlled by terahertz time domain spectroscopy), is considered in 


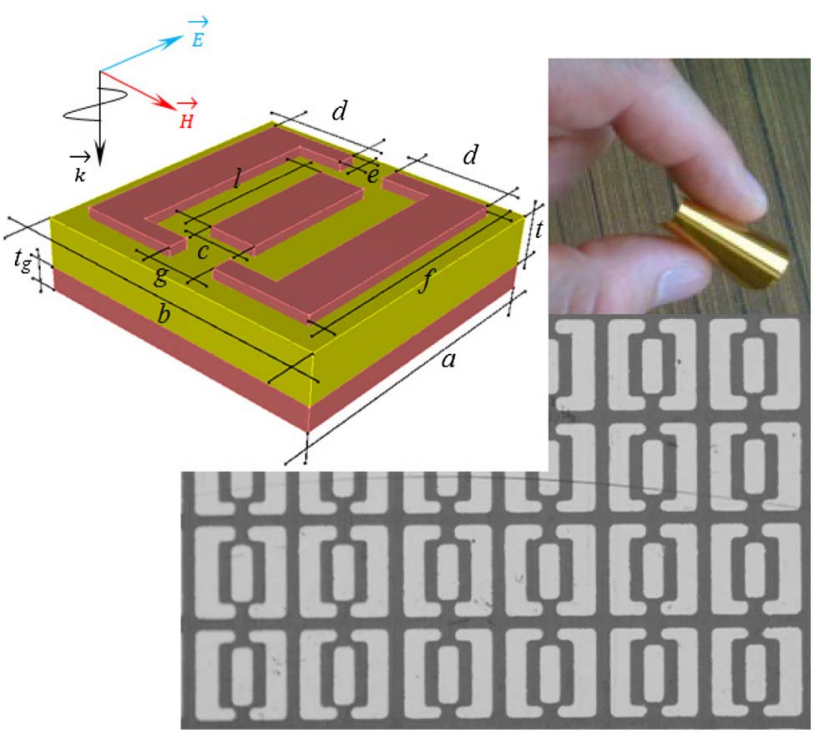

Fig. 1. Microscope image of the proposed metamaterial absorber. The relevant geometrical dimensions of the elemen tary meta cell viewed in the inset (top left) of the figure are $a=b=300 \mu \mathrm{m} ; \quad c=50 \mu \mathrm{m} ; \quad d=100 \mu \mathrm{m} ; \quad e=20 \mu \mathrm{m} ; \quad f=$ $250 \mu \mathrm{m} ; g=50 \mu \mathrm{m} ; l=150 \mu \mathrm{m} ; t=50 \mu \mathrm{m} ; t g=200 \mu \mathrm{nm}$. In the inset (top right) of the figure, we can see the dielectric substrate used as a spacer caught between two fingers in order to illustrate its large flexibility.

simulation and used in the manufacturing process. Due to its low thickness, the chosen dielectric spacer has good mechanical flexibility (see the inset top right of Fig. 1), which is suitable for conformal geometry applications such as radar absorbers covering the fuselage of military aircraft. Standard optical lithography relying on clean room and microelectronics facilities was used to fabricate our experimental prototype. Note that an infinite structure was considered in simulation along the $\mathrm{E}$ and $\mathrm{H}$ fields, while a prototype covering a more realistic surface of about $2 \times 2 \mathrm{~cm}^{2}$ was fabricated.

We performed simulations based on the finite element method to predict the spectral response of the structure. The sample is illuminated by a plane wave with TM polarization ( $E \|$ cut wires) at normal incidence with only one layer along the direction of propagation $k$. Terahertz time domain spectroscopy based on a TPS3000 setup equipped with an external gantry reflection module covering a wide frequency range up to $3 \mathrm{THz}$ in reflection configuration and up to $4 \mathrm{THz}$ in transmission configuration was used to characterize the response of the metamaterial absorber.

In the transmission measurements, the incident plane waves are normal to the sample surface, and the transmitted $\mathrm{THz}$ electric field is normalized with respect to transmission in free space between the emitter and the receiver. Similarly, the reflection of the terahertz electric field measured for the sample is normalized using a sheet of metal as a reflecting mirror. A good qualitative agreement is reported between the simulated (solid lines) and the measured (dashed lines) reflection and absorption spectra [see Fig. 2(a)]. The absorption coefficient $A(\omega)$ is obtained by $A(\omega)=1-T(\omega)-R(\omega)$, where $T(\omega)$ is equal to zero, due to the $200 \mathrm{~nm}$ thick aluminum used as a ground plane on the back face of the Kapton substrate. In our case, we emphasize the use of singlepattern elementary cells to achieve multiple absorption peaks. One can observe three main absorption peaks around frequencies of $0.2,0.46$, and $0.75 \mathrm{THz}$, with levels of about $45 \%, 85 \%$, and $97 \%$, respectively, in simulation and about $80 \%$ in measurement. In order to attempt to understand the origin of these peaks, we performed further numerical simulations. Our investigations reveal that each peak corresponds in fact to the contribution of different sub-elements constituting the unit cell of the structure; thus the overall absorption spectrum is the superposition of each sub-element's contribution. The first absorption peak that appears around $0.22 \mathrm{THz}$ comes from the two-gap SRRs, while the second one at $0.46 \mathrm{THz}$ corresponds to the contribution of the cut-wires. The third absorption peak occurring at about $0.75 \mathrm{THz}$ is present in the absorption spectrum of both SRRs and cut wires [see Fig. 2(b)]. One can observe a slight frequency shift between simulation and experiment at the second absorption peak, arising from the cut wires ( $0.46 \mathrm{THz}$ in simulation, and $0.48 \mathrm{THz}$ in measurement). Simulations performed (but not shown here), taking into account different lengths $l$ of the cut wires around the nominal length $l=150 \mu \mathrm{m}$, showed a drastic impact on the frequency shift [e.g., when $l=100 \mu \mathrm{m}, f$ (cut wires) $=$ $0.64 \mathrm{THz}$, when $l=190 \mu \mathrm{m}, f($ cut wires $)=0.3 \mathrm{THz}$, when $l=146 \mu \mathrm{m}, \quad f$ (cut wires) $=0.48 \mathrm{THz}, \quad$ which perfectly matches with the measured frequency]. Nevertheless, although there are minor differences in amplitude and bandwidth of the resonances due to manufacturing imperfections, the reflection and absorption spectra obtained from measurements confirm well the trend obtained from simulations.

By considering an alternative double-layered metamaterial absorber (DLMA) along the direction of propagation $k$, the amplitude of the first absorption peak occurring around $0.22 \mathrm{THz}$ can be enhanced. The elementary cell of the proposed DLMA is represented in Fig. 3(a). The bottom face of the first layer entirely

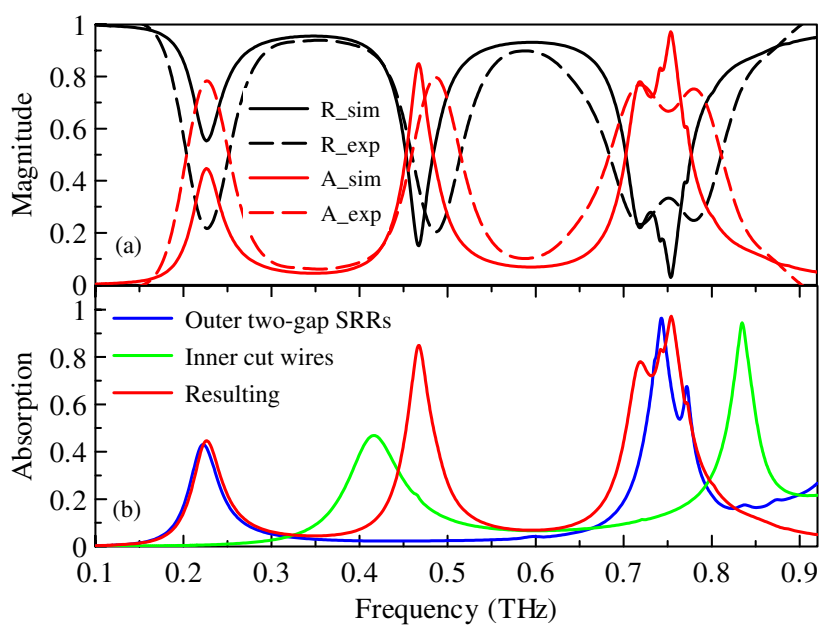

Fig. 2. (a) Simulated (solid lines) and measured (dashed lines) reflection and absorption spectra of the triple band metamate rial absorber. (b) Calculated absorption of the entire structure and that resulting from the contribution of different sub elements constituting the unit cell of the metamaterial based absorber. 


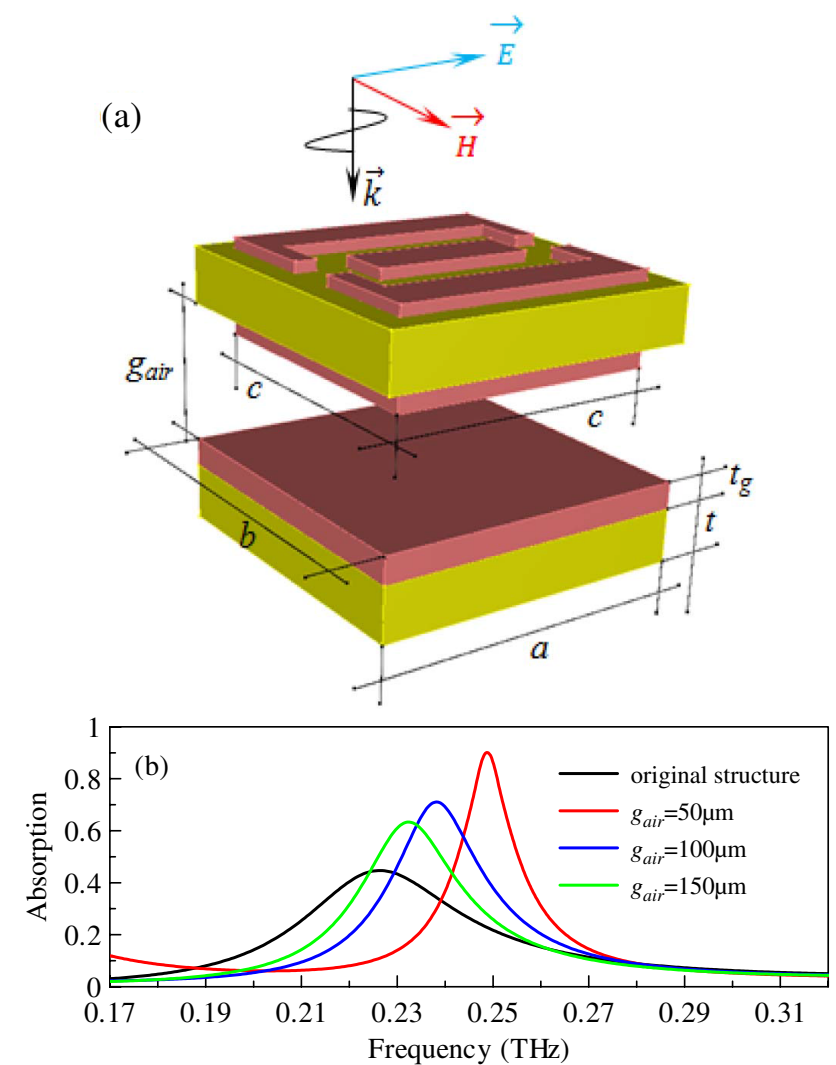

Fig. 3. (a) Elementary cell of the DLMA proposed as an alter native to the original structure in order to enhance the ampli tude of the fundamental absorption peak, with the relevant geometrical dimensions: $a=b=300 \mu \mathrm{m} ; c=250 \mu \mathrm{m} ; g_{a i r}$ is varying from 50 to $150 \mu \mathrm{m} ; t g=200 \mathrm{~nm} ; t=50 \mu \mathrm{m}$. (b) Optimi zation of the design parameter $g_{\text {air }}$ for the best compromise in amplitude of the fundamental peak absorption initially occurs around $0.22 \mathrm{THz}$.

coated with $200 \mathrm{~nm}$ thick aluminum, serving initially as a ground plane, is replaced by an array of square patches of $250 \mu \mathrm{m} \times 250 \mu \mathrm{m}$. The new ground plane is printed on the top side of a second layer separated by an air gap $g_{\text {air }}$ from the first layer, as represented in Fig. 3(a). Upon increasing the air gap between the layers, the following statements can be made: (1) the level of the first absorption peak around the frequency of $0.22 \mathrm{THz}$ is enhanced as shown in Fig. 3(b). We propose an optimum air gap of $g_{\text {air }}=50 \mu \mathrm{m}$, which leads to an absorption peak with $88 \%$ of amplitude at about $0.25 \mathrm{THz}$. (2) The increase of the air gap is also accompanied by a red shift of the fundamental absorption peak, due to the increase in the optical thickness of the structure along the direction of the propagation $k$. (3) The positions and amplitudes (not shown here) of the second and third absorption peaks occurring at frequencies 0.46 and $0.75 \mathrm{THz}$, respectively, are not significantly affected by the new configuration of the metamaterial absorber.

In summary, we have designed, fabricated, and experimentally demonstrated a thin-flexible terahertz multiband (three main peaks) metamaterial-based absorber operating with TM polarized radiation at normal incidence. A maximum absorption level of about $96 \%$ is achieved in simulation and about $80 \%$ in experiments. This is an encouraging step toward many promising applications such as filtering, antennas, and stealth systems.

\section{References}

1. V. G. Veselago, Sov. Phys. Usp. 10, 509 (1968).

2. R. A. Shelby, D. R. Smith, and S. Schultz, Science 292, 77 (2001).

3. J. B. Pendry, A. J. Holden, D. J. Robbins, and W. J. Stewart, J. Phys. Condens. Matter 10, 4785 (1998).

4. J. B. Pendry, A. J. Holden, D. J. Robbins, and W. J. Stewart, IEEE Trans. Microwave Theor. Tech. 47, 2075 (1999).

5. T. Maier and H. Brueckl, Opt. Lett. 35, 3766 (2010).

6. X. Liu, T. Starr, A. F. Starr, and W. J. Padilla, Phys. Rev. Lett. 104, 207403 (2010).

7. N. Landy, C. M. Bingham, T. Tyler, N. Jokerst, D. R. Smith, and W. J. Padilla, Phys. Rev. B 79, 125104 (2009).

8. N. I. Landy, S. Sajuyigbe, J. J. Mock, D. R. Smith, and W. J. Padilla, Phys. Rev. Lett. 100, 207402 (2008).

9. H. Tao, C. M. Bingham, D. Pilon, K. Fan, A. C. Strikwerda, D. Shrekenhamer, W. J. Padilla, X. Zhang, and R. D. Averitt, J. Phys. D 43, 225102 (2010).

10. X. Shen, T. J. Cui, J. Zhao, H. F. Ma, W. X. Jiang, and H. Li, Opt. Express 19, 9401 (2011).

11. R. Yahiaoui, H. Němec, P. Kužel, F. Kadlec, C. Kadlec, and P. Mounaix, Opt. Lett. 34, 3541 (2009).

12. H. Wakatsuchi, S. Greedy, C. Christopoulos, and J. Paul, Opt. Express 18, 22187 (2010).

13. Y. Q. Ye, Y. Jin, and S. He, J. Opt. Soc. Am. B 27, 498 (2010).

14. K. B. Alici, A. B. Turhan, C. M. Soukoulis, and E. Ozbay, Opt. Express 19, 14260 (2011).

15. R. Yahiaoui, U. Chung, C. Elissalde, M. Maglione, V. Vigneras, and P. Mounaix, Appl. Phys. Lett. 101, 042909 (2012).

16. H. Li, L. H. Yuan, B. Zhou, X. P. Shen, Q. Cheng, and T. J. Cui, J. Appl. Phys. 110, 014909 (2011).

17. G. Dayal and S. A. Ramakrishna, J. Opt. 15, 055106 (2013).

18. D. Wen, H. Yang, Q. Ye, M. Li, L. Guo, and J. Zhang, Phys. Scr. 88, 015402 (2013).

19. R. Singh, I. A. I. Al Naib, Y. Yang, D. R. Chowdhury, W. Cao, C. Rockstuhl, T. Ozaki, R. Morandotti, and W. Zhang, Appl. Phys. Lett. 99, 201107 (2011).

20. Y. Yang, R. Huang, L. Cong, Z. Zhu, J. Gu, Z. Tian, R. Singh, S. Zhang, J. Han, and W. Zhang, Appl. Phys. Lett. 98, 121114 (2011). 